\title{
SELECTED MORPHOTIC PARAMETERS DIFFERENTIATING ULCERATIVE COLITIS FROM CROHN'S DISEASE
}

\author{
Anna KASPERCZUK* \\ ${ }^{*}$ Faculty of Mechanical Engineering, Institute of Biomedical Engineering, \\ Bialystok Technical University, ul. Wiejska 45C, 15-351 Bialystok, Poland \\ a.kasperczuk@pb.edu.pl
}

received 22 May 2020, revised 20 September 2021, accepted 24 September 2021

\begin{abstract}
This paper presents a method that binds statistical and data mining techniques, which aims to support the decision-making process in selected diseases of the digestive system. Currently, there is no precise diagnosis for ulcerative colitis (UC) and Crohn's disease (CD). Specialist physicians must exclude many other diseases occurring in the colon. The first goal of this study is a retrospective analysis of medical data of patients hospitalised in the Department of Gastroenterology and Internal Diseases, Bialystok, and finding the symptoms differentiating the two analysed diseases. The second goal is to build a system that clearly points to one of the two diseases UC or $C D$, which shortens the time of diagnosis and facilitates the future treatment of patients. The work focuses on building a model that can be the basis for the construction of action rules, which are one of the basic elements in the medical recommendation system. Generated action rules indicated differentiating factors, such as mean corpuscular volume, platelets (PLTs), neutrophils, monocytes, eosinophils, basophils, alanine aminotransferase (ALAT), creatinine, sodium and potassium. Other important parameters were smoking and blood in stool.
\end{abstract}

Keywords: action rule, data mining, colon disease, morphotic parameters, smoking

\section{INTRODUCTION}

Inflammatory bowel diseases (IBDs) are diseases in the course of which, there is chronic inflammation of the gastrointestinal tract. Their conditions are not fully understood, but it is known that immunological, genetic and environmental factors play a role in the pathogenesis of the disease state (Cappello and Morreale, 2016; Crohn et al., 1932). It is possible that the onset of the disease is induced by reactions between these factors. Some sources say that allergic factors, as well as bacterial and viral infections, are also responsible (Crohn et al., 1932). The group of IBDs includes Crohn's disease (CD) and ulcerative colitis (UC) (Cappello and Morreale, 2016). Despite the fact that they are two separate diseases, their differentiation is difficult for clinicians and requires careful analysis of clinical symptoms, endoscopic and radiological examinations and the histopathological picture. Sometimes, it is not possible to classify a case under any of these diseases. This situation occurs in about $10 \%-15 \%$ of diagnosed patients. Then, they are classified into the group indeterminate colitis (Daniluk et al., 2017).

It is extremely important to understand the greater number of UC and CD differentiating factors, as well as the relationships between the parameters, which will contribute to faster diagnosis of new patients and thus improve the quality of diagnosis and treatment (Daniluk et al., 2017).

\subsection{Features of $C D$}

$C D$ is a chronic inflammatory disease that can affect any part of the digestive tract. Clinical symptoms most often depend on the location of the inflammatory changes. Literature indicates abdominal pain (most often in the right hip), gas, weight loss, weakness and fever. Other observed symptoms are perianal abscesses. Inflammatory changes in the digestive tract are discontinuous. Additionally, apart from the mucosa, inflammatory changes also affect all other parts of the intestinal wall (Crohn et al. 1932; Dolapcioglu et al., 2014; Kirsner, 1988).

\subsection{Features of UC}

UC is a chronic inflammatory disease. Symptoms include frequent diarrhoea with blood or mucus. Fistulas and abscesses are rare. Permanent inflammatory changes are located along the entire length of the large intestine in the form of superficial ulcers. The histological picture shows granulocytes and lymphocytic infiltrates of the mucosa. In the advanced stage, i.e. after about 10 years of the disease, changes in epithelial structure in the form of dysplasia may appear, leading to the formation of tumours (Daniluk et al., 2017; Priyamvada et al., 2015).

The development of medicine has contributed to the possibility of using laboratory tests to assess the burden of IBDs, as symptom-based results are too subjective to predict the treatmentoptions properly and to calculate the risk of relapse. In the present study, we were searchedfor features to distinguish $C D$ from UC easily. Laboratory tests are helpful in assessing the activity of each disease. Morphological tests of blood and determination of various biochemical parameters allow the early detection of changes and side effects of therapy. 


\section{MATERIALS AND METHODS}

\subsection{Data Collection}

The data used in this study were obtained on patients of the Department of Gastroenterology and Internal Diseases of the Medical University of Bialystok Clinical Hospital. The data acquisition process consisted of interviewing patients with IBD and analyzing their medical records. The patients were diagnosed based on clinical symptoms, morphological, and radiological, endoscopic and histological findings.

Information on the following laboratory results was collected: white blood cells (WBCs) $\left[\times 10^{3} / \mu \mathrm{L}\right]$, red blood cells (RBCs) $\left[\times 10^{6} / \mu \mathrm{L}\right]$, mean corpuscular volume (MCV) [fL], platelets (PLTs) $\left[\times 10^{3} / \mu \mathrm{L}\right]$, neutrophils $\left[\times 10^{3} / \mu \mathrm{L}\right]$, lymphocytes $\left[\times 10^{3} / \mu \mathrm{L}\right]$, monocytes $\left[\times 10^{3} / \mu \mathrm{L}\right]$, eosinophils $\left[\times 10^{3} / \mu \mathrm{L}\right]$, basophils $\left[\times 10^{3} / \mu \mathrm{L}\right]$, glucose $[\mathrm{mg} / \mathrm{dL}]$, bilirubin $[\mathrm{mg} / \mathrm{dL}]$, aspartate aminotransferase (AspAT) [lU/L], alanine aminotransferase (ALAT) [IU/L], amylase [IU/L], prothrombin time (PT) [sec], international normalised ratio (INR), fibrinogen $[\mathrm{mg} / \mathrm{dL}]$, urea $[\mathrm{mg} / \mathrm{dL}]$, creatinine $[\mathrm{mg} / \mathrm{dL}]$, sodium $[\mathrm{mmol} /$ $\mathrm{L}]$, potassium [mmol/L] and C-reactive protein (CRP) $[\mathrm{mg} / \mathrm{dL}]$. Sociodemographic data were also taken into account: age, gender, smoking (a smoker is a patient who smoked at least a year without interruption). In addition, symptoms of medical conditions such asblood in the stool and a palpable tumour within the abdominal cavity, were taken into account.

\subsection{Feature Selection}

The selection of features was performed using statistical methods. The Mann-Whitney test was used for comparison of the CD group with the UC group in the case of quantitative data, if the parameters were not shown to be in normal distribution; Student's $t$-test was used if there was compatibility with normal distribution and homogeneity of variance; and Cochran-Cox test was used if compliance with the normal distribution was shown but there was no homogeneity of variance. In the case of comparison of data on the qualitative scale, a chi-square test was used. The ShapiroWilk test was used to check compliance with the normal distribution, and the Leven test was used to test homogeneity of variance. The significance level was assumed as $a=0.05$. Selected features were used because of the construction of classifiers using three algorithms of knowledge extraction. For the machine learning algorithms, the cross-validation method was used. It involves the division of the studied statistical sample into subsets: the training and the test sets. The analyses are carried out on the training set, while the test set is used to confirm the reliability of the obtained results.

\subsection{Action Rules Mining}

Many scientific studies show that the use of post-field methods in the analysis of medical data gives opportunities to improve the quality of diagnoses, that too at an early stage (Bebas et al., 2021). This work focuses on using action rule extraction methods.
Let us assume that $\mathrm{S}=(\mathrm{X}, \mathrm{A}, \mathrm{V})$ is an information system, where $\mathrm{X}$ is a non-empty, finite set of objects, $\mathrm{A}$ is a non-empty, finite set of attributes, $V$ is a set of all attributes' values (Dardzinska, 2014; Ras and Dardzinska, 2011). Then, a : X $\rightarrow$ $V_{a}$ is a function for any a $\in A$, that returns the value of the attribute of a given object. The set of attributes can be divided into three subsets $A=A_{1} \cup A_{2} \cup D$. Here, $A_{1}$ is a set of stable attributes, $A_{2}$ is a set of flexible attributes and the set of decision attributes is described by D (Dardzinska, 2014; Han and Kamber, 2006; Dardzinska and Kasperczuk; 2018; Dardzinska and Rpmaniuk, 2016). By stable attributes, we mean attributes with unchangeable values (e.g. age, gender), while for the flexible attributes, the values can be changed (e.g. blood pressure, weight, haemoglobin level). Information systems can be also seen as decision tables with one stable attribute, flexible attributes and the decision attribute $d$ (Gürdal and Dardzinska, 2017; Kasperczuk and Dardzinska, 2019).

Our goal is to find links between the classification and decision attributes. Classification attributes can be represented as measurements, parameters from the patient's test results, personal data, and so on. The decision is a value related to the acquired knowledge, e.g. given directly by an expert or from observation, such as the diagnosis or treatment made. The decision allocated objects from the decision system, thus creating an easy reference model. Then, the acquired knowledge about the relationships between the classification and decision-making attributes will be used to create action rules that will allow us to find the differences between the two decisions.

The modified action rules algorithm in incomplete system (MARAIS) was developed to support the extraction of knowledge from medical information systems, taking into account the specificity of the data contained in them using the conviction measure. MARAIS is an extension of the well-known action rules discovery based on agglomerative strategy (ARAS) algorithm. Using the created algorithm, classification rules were built and action rules were extracted next. From the set of action rules, the knowledge base was created, which was subjected to qualitative analysis. All the rules were characterised by an extremely high level of support and trust and a sufficiently low value of the belief measure. Based on the acquired knowledge, the symptoms differentiating UC and CD were found. Action rules are logical terms defining knowledge for desirable actions related to the hidden objects in a database. The intent here is to concentrate on objective measures for actionability, which is defined as the extent to which a user can gain benefits from the discovered patterns, such as in the medical domain $[10,17]$. Suppose an actionable goal of $r=[\omega *(\alpha \rightarrow \beta) \rightarrow(\theta \rightarrow \psi)]$, where $\omega, \alpha, \beta, \theta$ and $\psi$ are descriptions of objects, e.g. in the case of patients, where $p$ is described as the satisfaction of a designed condition and the changeable measure of $(\alpha \rightarrow \beta)$ for patients who are registered in a database with the expected result $(\theta \rightarrow \psi)$. There are two conceivable perspectives in terms of the strategies of actionability. One is the constituent of post-field analysis at the back end of the knowledge discovery system (Dardzinska, 2013). This approach does not utilise the prior knowledge of the expert systems to lead the rule-generation process, which is purely subjective. The other approach is solely objective. It implements the input knowledge of the domain to control the rule generation process, which leads to determination of instrumental knowledge and compares it with some standard beliefs. In this paper, we concentrate on the object-driven 
approaches of actionability. Object-driven patterns can be generated straightforward from the dataset and then implemented for the final outcome (Dardzinska, 2013; Ras and Dardzinska, 2011). By object-driven action rule $r$ in an information system $S$, the expression can be represented as follows:

$\mathrm{r}=[[(\mathrm{a} 1=\omega 1) *(\mathrm{a} 2=\omega 2) * \ldots *(\mathrm{aq}=\omega \mathrm{q})] *$

$(\mathrm{b} 1, \alpha 1 \rightarrow \beta 1) *(\mathrm{~b} 2, \alpha 2 \rightarrow \beta 2) * \ldots *(\mathrm{bp}, \alpha \mathrm{p} \rightarrow$

$\beta \mathrm{p})] \rightarrow[(\mathrm{d}, \mathrm{k} 1 \rightarrow \mathrm{k} 2)]$,

where $\{b 1, b 2, \ldots, b p\}$ are flexible and $\{a 1, a 2, \ldots, a q\}$ are stable attributes in $S$. Further, it is assumed that $\omega i \in$ $\operatorname{Dom}(a i), i=1,2, \ldots, q$ and $\alpha \mathrm{i}, \beta \mathrm{i} \in \operatorname{Dom}(\mathrm{bi}), \mathrm{i}=1,2, \ldots, \mathrm{p}$. When $(a i=\omega i)$, the value of the attribute becomes ai and is equal to $\omega \mathrm{i}$, and (bj, $\alpha \mathrm{j} \rightarrow \beta \mathrm{j}$ ); it shows that value of the attribute bj has been changed from $\alpha j$ to $\beta j$. That is to say, object $x \in S$ supports an action rule $r$ in $S$, if there is an object $y \in S$ such that:

$(\forall \mathrm{i} \leq \mathrm{p})[[\mathrm{bi}(\mathrm{x})=\alpha \mathrm{i}] *[\mathrm{bi}(\mathrm{y})=\beta \mathrm{i}]],(\forall \mathrm{i} \leq \mathrm{q})[\mathrm{ai}(\mathrm{x})=$ $\mathrm{ai}(\mathrm{y})=\omega \mathrm{i}], \mathrm{d}(\mathrm{x})=\mathrm{k} 1$ and $\mathrm{d}(\mathrm{y})=\mathrm{k} 2$.

The aforementioned object-driven perspective induces a set of structures that are implemented mathematically to evaluate a dataset. By implementing the objective approach with action rules, some of the chosen objects may be reclassified from one stage to another by modifying some of the relevant flexible attributes. In a previous paper (Dardzinska and Kasperczuk, 2019), we suggested and formulated a simple rule extraction algorithm to build the action rules of a single classification rule, which we named ARAS. It is a bottom-up approach in a breath-first manner to form all frequent item sets with a qualified part of length $k$, before forming those qualified parts of length $k+1$. More information on the application domain of an experiment of ARAS is available elsewhere (Dardzinska, 2013; Ras and Dardzinska, 2011).

\section{RESULTS}

In the experiment, we used the data of patients suffering from UC (86 cases) and CD (66 cases). The analysis was based on the construction of action rules and their application to real medical data. We discretised the data of the selected patients and extracted the highest related attributes in test values. Then, we validated the results along with the patients' history and physical examination results. The results are very promising. More than $95 \%$ of the patients were correctly reclassified.

The study group consisted of individuals with $U C(N N=86$, women $N=32$, men $N=54)$, and patients with $C D(N=66$, women $\mathrm{N}=32$, men $\mathrm{N}=34$ ) were diagnosed.

The age of the patients in the study group was $38.05 \pm 16.57$ years, with the average age of women being $35.97 \pm 15.56$ years and that of men being $39.57 \pm 17.19$ years. The mean age in the CD group was $34.42 \pm 14.30$ years (mean age of women: $36.19 \pm 16.90$ years; men: $32.76 \pm 11.34$ years). The mean age in the group of UC patients was $40.84 \pm 17.70$ years (mean age of women: $35.75 \pm 14.37$ years; men: $43.85 \pm 18.88$ years).

Modelling pointed to variables that are significantly different in the analysed groups. Among the biochemical parameters in the blood tests, MCV $(p=0.013)$, PLTs $(p=0.018)$, neutrophils $(p=0.043)$, monocytes $(p=0.033)$, eosinophils $(p=0.003)$, basophils $(p=0.001)$, ALAT $(p=0.002)$, creatinine $(p=0.017)$, sodium $(p<0.001)$ and potassium $(p=0.018)$ were all-significant.
Other important parameters were smoking $(p<0.001)$ and blood in stool $(p<0.001)$.

A number of rules were generated, but only those whose level of support, trust and conviction exceeded those assumed were accepted. From the set of rules extracted using the MARAIS algorithm, the following selected set of action rules was generated:

1. Creatinine $<0.69 A N D$ blood in stool $=$ $0 A N D$ smoking $=0$ AND $(P L T<524.5 \Rightarrow P L T \geq$ $524.5) \Rightarrow(U C \Rightarrow C D)$

Support: 0.140, Confidence: 1.0, Conviction: 0.03

2. Creatinine $<0.69 A N D$ blood in stool $=$ 1 AND (smoking $=0 \Rightarrow$ smoking $=1) \Rightarrow$ $(U C \Rightarrow C D)$

Support: 0.159, Confidence: 1.0, Conviction: 0.11

3. Creatinine $\geq 0.69$ AND smoking $=$ 0 AND potassium $\geq 4.12$ AND $M C V<$ 85.73 AND PLT $\geq 273$ AND (Neutrophils < $16.22 \Rightarrow$ Neutrophils $\geq 16.22) \Rightarrow(U C \Rightarrow C D)$

Support: 0.156, Confidence: 1.0, Conviction: 0.19

4. Creatinine $\geq 0.69$ AND smoking $=$ 0 AND potassium $\geq 4.12$ AND $M C V \geq$ 89.85 AND Creatinine $<0.81$ AND $(M C V \geq 95.5 \Rightarrow$ $M C V<95.5) \Rightarrow(U C \Rightarrow C D)$

Support: 0.143 , Confidence: 1.0, Conviction: 0.02

5. Creatinine $\geq 0.76$ AND smoking $=$ 1 AND sodium $\geq 137$ AND Eosinophils $\geq$ 0.33 AND sodium $<138.52$ AND $(M C V \geq$ $83.68 \Rightarrow M C V<83.68) \Rightarrow(U C \Rightarrow C D)$

Support: 0.154, Confidence: 1.0, Conviction: 0.17

6. Creatinine $<0.69 A N D$ blood in stool $=$ 0 AND smoking $=0$ AND $(P L T \geq 524.5 \Rightarrow$ $P L T<524.5) \Rightarrow(U C \Rightarrow C D)$

Support: 0.103, Confidence: 1.0, Conviction: 0.12

7. Creatinine $<0.69$ AND blood in stool $=$ 1 AND (smoking $=0 \Rightarrow$ smoking $=1) \Rightarrow$ $(U C \Rightarrow C D)$

Support: 0.112, Confidence: 1.0, Conviction: 0.19

\section{DISCUSSUION}

The characteristics of UC and CD are often ambiguous. and their diagnosis creates many problems that are difficult to overcome (Daniluk et al. 2017). In diagnosis, it is important to make a quick yet accurate diagnosis. Therefore, it is necessary to look for symptoms that directly differentiate the disorders. This will make it possible to build recommendation systems for specialists, accelerate diagnosis and improve the level of medical services.

The constructed action rules indicate the features that, upon change, cause a transition to another group, thus emphasising the differences between the values of the variables in the analysed groups. Action rules extraction was performed using the MARAIS algorithm. All the rules were characterised by an extremely high level of support and trust, as well as a sufficiently low value of the measure of belief, which - at that time - indicated that the values 
of the attributes making up the rule were related. The constructed rules indicate parameters that differ significantly in the indicated groups. These include smoking, PLT levels, neutrophil levels and MCV levels. Additionally, they show quantitative differences concerning the levels of the mentioned parameters in the two analysed groups.

Analysis indicated that people who were diagnosed with UC did not smoke in most cases $(\mathrm{N}=76)$. The number of smokers $(\mathrm{N}$ $=48$ ) in relation to non-smokers ( $N=18)$ was significantly higher among patients with $C D$. This indicates a lower risk of $U C$ in the smoking group. The results obtained are confirmed by the literature (Daniluk et al. 2017).

The exact values of the blood parameters that differentiate UC and $C D$ are not known in the literature. Scientists only mention likely differences, but quantitative data is not known (Dolapcioglu et al., 2014). This article shows what levels of significantly different factors determine the occurrence of $U C$ or $C D$.

$C D$ and $U C$ are characterised by the dispersed accumulation of lymphocytes in the intestinal mucosa. Lymphocytes are cells of the immune system that belong to the granulocytes that are involved in and underlie the immune response. The literature confirms changes in their number in UC and CD patients, but specific levels are not known. It is important to know whether there are any differences in the level of this parameter between UC and CD (Giuffrida et al., 2018; Sarfati et al., 2015; Priyamvada et al., 2015).

Research indicates that eosinophils play a role in the pathogenesis of IBDs. Laboratory examinations revealed the accumulation and activation of eosinophils in the active inflammatory intestinal mucosa in patients with UC and CD. However, there is a lack of accurate quantitative data and their possible distinction in the two diseases analysed in this paper (Kasperczuk et al., 2019; Merigo et al., 2018). Studies indicate that platelet count (PLT) often increases in association with active inflammation, and therefore may be a differentiating factor between $U C$ and $C D$.

Another blood morphotic parameters reported in the literature are creatinine and urea. Their levels can change in IBDs. Unfortunately, differences in levels of this important parameter between UC and CD are not exactly known (Daniluk et al., 2017).

Additionally, changes in electrolyte absorption in diarrhea are common in IBD. Therefore, this study investigated the differences in sodium and potassium levels in UC and $C D$, In IBD, changes in the absorption of electrolytes in diarrhoea are frequent. Therefore, the study examined differences in sodium and potassium levels in UC and CD to see whether they are significantly different (Shiffer et al., 2017; Yazici et al., 2010; Zho and Liu, 2017).

Patients in the two analysed groups show significantly different PLT levels. If creatinine is maintained at the level mentioned in the rule, there is no blood in the faeces and the patient is not a smoker, then a PLT level $\geq 524.5 \times 10^{\wedge} 3 / \mu \mathrm{L}$ will be characteristic of $C D$ and levels lower than that level will indicate UC.

If the patient's creatinine level is $<0.69 \mathrm{mg} / \mathrm{dL}$, there is no blood in the faeces and the person is a smoker, the patient will be diagnosed with $C D$. This indicates that while maintaining this level of creatinine and with the occurrence of blood in the faeces, smoking will indicate $\mathrm{CD}$; otherwise, the patient will belong to the UC group.

The parameter differentiating these diseases is the level of neutrophils in the blood. If the patient has the creatinine, potassium, MCV and PLT levels specified in the rule but is not a smoker, a neutrophil value $<16.22 \times 10^{\wedge} 3 / \mu \mathrm{L}$ will be characteristic of $C D$ and a value $\geq 16.22 \times 10^{\wedge} 3 / \mu \mathrm{L}$ is indicative of $U C$.

For both diseases, while maintaining levels of other parameters, such as creatinine, potassium and smoking, the MCV level may exceed $89.95 \mathrm{fL}$; values $>95.5 \mathrm{fL}$ indicate $\mathrm{UC}$, while levels between $89.85 \mathrm{fL}$ and $95.5 \mathrm{fL}$ are characteristic of CD,.

If the patient's creatinine level is maintained at a level $\geq 0.76$ $\mathrm{mg} / \mathrm{dL}$, the patient is a smoker, the sodium is maintained at 137$138.52 \mathrm{mmol} / \mathrm{L}$, the eosinophil count is $\geq 0.33 \times 10^{\wedge} 3 / \mu \mathrm{L}$ and the $\mathrm{MCV}$ is $\geq 83.68 \mathrm{fL}$, the patient will be assigned to the CD group.

While maintaining appropriate values of parameters and characteristics (the patient's creatinine level is kept $<0.69 \times 10^{\wedge} 3$ $/ \mu \mathrm{L}$, no blood is found in the faeces, the person is a non-smoker), when the PLT value exceeds $524.5 \times 10^{\wedge} 3 / \mu \mathrm{L}$, patients have UC; otherwise, they are classified as having $C D$.

While maintaining an adequate level of creatinine $(<0.69 \times 10$ $\wedge 3 / \mu \mathrm{L}$ ) and in the presence of blood in the faeces, smoking will cause the person to be classified as suffering from CD; otherwise, the classification will be UC.

\section{CONCLUSION}

In medical and biological systems, the operating rules show very promising results: the physician can investigate the effect of the choice of treatment on the patient's condition. In addition, the rules of operation indicate those features that cause a transition to another group when they are changed, thus emphasising the differences between the values of the variables in the analysed groups. Research shows new values of important parameters that differentiate the IBDs, such as MCV, PLTs, neutrophils, monocytes, eosinophils, basophils, ALAT, creatinine, sodium and potassium. Other important parameters were smoking and blood in stool. This is extremely important because it can accelerate the diagnostic process of IBD patients.

\section{REFERENCES}

1. Bebas E., Borowska M., Derlatka M., Oczeretko E., Hladunski M., Szumowski P., Mojsak M. (2021) Machine-learning-based classification of the histological subtype o non-small-cell lung cancer using MRI texture analysis. Biomedical Signal Processing and Control, vol. 66, 1-6.

2. Cappello, M.; Morreale, G.C. (2016) The Role of Laboratory Tests in Crohn's Disease. Clin Med Insights Gastroenterol, 9, 51-62.

3. Crohn B.B., Ginzburg L., Oppenheimer G.D. (1932) Regional ileitis. A pathologic and clinical entity, J Am Med Ass. 99:1323-1329.

4. Daniluk J, Daniluk U, Reszec J, Rusak M, Dabrowska M, Dabrowski A.(2017) Protective effect of cigarette smoke on the course of dextran sulfate sodium-induced colitis is accompanied by lymphocyte subpopulation changes in the blood and colon. Int $\mathrm{J}$ Colorectal Dis, 32, 1551-1559.

5. Dardzinska A. (2013), Action Rules Mining. Springer, pp. 90.

6. Dardzinska A., Kasperczuk A. (2018), Decision-making Process in Colon Disease and Crohn's Disease Treatment, Acta Mechanica et Automatica, Vol. 12 no. 3, pp. 227-231.

7. Dardzinska A., Romaniuk A. (2016), Mining of Frequent Action Rules, Machine Intelligence and Big Data in Industry: 6th International Conference on Pattern Recognition and Machine Intelligence, 87-95

8. Dolapcioglu, C.; Soylu, A.; Kendir, T.; Ince, A.T.; Dolapcioglu, H.; Purisa, S.(2014) Coagulation parameters in inflammatory bowel disease. Int J Clin Exp Med , 7, 1442-1448. 
9. Giuffrida, P.; Corazza, G.R.; Di Sabatino, A. (2018) Old and New Lymphocyte Players in Inflammatory Bowel Disease. Dig Dis Sci, 63, 277-288.

10. Gren, S.T.; Grip, O. (2016) Role of Monocytes and Intestinal Macrophages in Crohn's Disease and Ulcerative Colitis. Inflamm Bowel Dis, 22, 1992-8.

11. Gürdal O., Dardzinska A. (2017), A New Approach to Clinical Medicine by Action Rules, International Journal of Development Research, 7(1), 11032-11039.

12. Han J., Kamber M. (2006), Data Mining: Concepts and Techniques, Morgan Kaufmann Publishers, Second Edition, 21-27.

13. Kasperczuk A, Daniluk J, Dardzinska A. (2019) Smart Model to Distinguish Crohn's Disease from Ulcerative Colitis. Appl. Sci, 9 , 1650.

14. Kasperczuk A. and Dardzinska A., (2016), Comparative Evaluation of the Different Data Mining Techniques Used for the Medical Database, Acta Mechanica et Automatica, Vol. 10 no. 3, pp. 233-238.

15. Kirsner J. B. (1988), Historical aspects of inflammatory bowel disease, J Clin Gastroenterol ,10:286-297.

16. Merigo, F.; Brandolese, A.; Facchin, S.; Missaggia, S.; Bernardi, P.; Boschi, F.; et al. (2018) Glucose transporter expression in the human colon. World J Gastroenterol, 24,775-793.

17. Priyamvada, S.; Gomes, R.; Gill, R.K.; Seksena, S.; Alrefai, W.A.; Dudeja, P.K. (2015) Mechanisms Underlying Dysregulation of Electrolyte Absorption in IBD Associated Diarrhea. Inflamm Bowel Dis, 21, 2926-2935.

18. Ras Z., Dardzinska A. (2011), From Data to Classification Rules and Action,. International Journal of Intelligent Systems, Wiley, 26(6), 572-590.
19. Sarfati, M.; Wakahara, K.; Chapuy, L.; Delespesse, G. ( 2015) Mutual Interaction of Basophils and T Cells in Chronic Inflammatory Diseases. Front Immunol, 6, 399.

20. Schieffer, K.M.; Bruffy, S.M.; Rauscher, R.; Koltun, W.A.; Yochum, G.S.; Gallagher, C.G. (2017) Reduced total serum bilirubin levels are associated with ulcerative colitis. PLoS One, 12, e0179267.

21. Yazici, A.; Senturk, O.; Aygun, C.; Celebi, A.; Caglayan, C.; Hulagu, S. (2010) Thrombophilic Risk Factors in Patients with Inflammatory Bowel Disease. Gastroenterology Res., 3, 112-119.

22. Zho, G.X.; Liu, Z.J. (2017) Potential roles of neutrophils in regulating intestinal mucosal inflammation of inflammatory bowel disease. J Dig Dis, 495-503.

Research was performed as a part of projects WZ/WM-IIB/2/2021 and financed with use of funds for science of MNiSW.

Anna Kasperczuk: (D) https://orcid.org/0000-0002-5919-5346 